\title{
Evidence-based medicine and psychiatry
}

\author{
John Geddes
}

Ir J Psych Med 1997; 14(3): 83-84

Key words: Evidence-based medicine (EBM); Psychiatry; Treatment outcome.

\section{What is EBM?}

Over the last five years the adjective 'evidence-based' has become difficult to avoid. Indeed, a MEDLINE search for articles containing the phrase 'evidence-based medicine' in their titles or abstracts reveals one mention in 1992 , rapidly increasing to 53 in 1996 . So great has been the increase that the National Library of Medicine now includes 'evidence-based medicine' as a $\mathrm{MeSH}$ heading for indexing papers.

But what is evidence-based medicine (EBM)? First and foremost, EBM is a set of strategies designed to help the clinician keep up-to-date and to base his clinical decision making on the best available external evidence. ${ }^{1}$ EBM has been espoused by policymakers, purchasers and others and, although the approach is open to misuse by these groups as a cost-cutting exercise, there are refreshing signs that they will be able to use the approach to help produce real improvements in patient care. ${ }^{2}$ However, the essential focus of EBM is on assisting doctors and other clinicians make decisions about individual patients. The steps involved in EBM include: a precise definition of the clinical problem (a crucial first step - in medical practice it will usually include making a diagnosis), an efficient search for the best available evidence, critical appraisal of the evidence and integration of the research findings with clinical expertise. Finally, the clinician assesses the outcome of the process and continues to improve his EBM skills.

At each stage of EBM, recent developments in information technology and clinical epidemiology and biostatistics are harnessed to make the process as rapid and as efficient as possible. A clear definition of the type of clinical problem is an essential prerequisite for determining the kind of evidence required and the best place to start looking for it. The aim of searching is to find the evidence which provides the least biased (and therefore most likely to be true) information.

For example, to answer a question about the best treatment for a particular problem, the clinician would look for a randomised controlled trial (RCT, or better still, a systematic review of randomised controlled trials) a study design which would be most likely to provide an unbiased estimate of the true treatment effect. ${ }^{3}$ The best place to start searching for a systematic review of RCTs is the Cochrane Library, which contains the Cochrane Database of Systematic Reviews (CDSR) and the Database of Abstracts of Reviews of Effectiveness (DARE)., ${ }^{4,5}$ The Cochrane Library is continually being added to and updated and is published quarterly on compact disk. If no usable review is found in the Cochrane Library or via MEDLINE, the clinician then looks for a single RCT. If no

John Geddes, MD, MRCPsych. Hon Consultant Psychiatrist and Director, Centre for Evidence-Based Mental Health, Department of Psychiatry, University of Oxford, Warneford Hospital, OXFORD OX3 7JX, England.

SUBMITTED: JULY 25, 1997. ACCEPTED: AUGUST 8, 1997. randomised evidence (systematic review or single RCT) is found, the clinician then looks for the best available nonrandomised evidence - recognising that it is more likely to provide a biased estimate of the effectiveness of the treatment. If, as occurs reasonably often, no usable external evidence is found, the clinician will need to base their decision on their clinical experience - but at least they have will have checked to see that there is nothing better!

After finding the best available evidence, the evidence needs to be critically appraised for its validity and usefulness (it's often helpful to use a standard checklist ${ }^{6,7}$ ). To help the application of the evidence to the clinical problem, it is usually helpful to translate the results into easily understandable indices such as the number need to treat a measure that clinicians find useful for assessing the effectiveness of treatments. ${ }^{8}$

\section{Why do we need EBM?}

From this description, it can be seen that EBM requires a number of skills (efficient searching of bibliographic databases, critical appraisal, numerical and statistical skills) which the average clinician may not have! So why should they embark on the laborious process of developing them? The main reason for using EBM is that clinical practice tends to lag behind research. This leads to helpful treatments not being used, harmful treatments being overused and widespread and unjustified variations in clinical practice. For example, in psychiatry, unexplained variations in practice have been reported in the use of ECT.' As Kendell has pointed out, variations in clinical practice for similar patient groups can only mean that there either is no evidence to allow clinicians to choose between treatments or that clinicians are not basing their practice on evidence. ${ }^{10}$ Claims that there is little evidence on which to base psychiatric practice are unfounded - the majority of common treatments can be based on evidence. ${ }^{11,12}$ The problem is more one of difficulties in accessing the evidence. EBM is aimed at overcoming these difficulties - by providing a coherent and comprehensive approach to narrowing the gap between research and practice.

\section{How to start using EBM in psychiatry}

The practice of EBM requires the acquisition of some new knowledge and skills and improved access to sources of evidence.

Probably the quickest way to get going is to read an introductory textbook,,$^{13.14}$ and to attend a workshop aimed at improving skills in both practicing and teaching EBM. Workshops on teaching EBM are now held regularly in the UK in Oxford and London and there is an annual introductory workshop on EBM for psychiatry held in Oxford.

One way to introduce EBM is to modify the nature of existing educational structures. For example, a journal club can be reorganised along EBM lines - this can be a useful way of introducing critical appraisal. ${ }^{15}$ At our journal club in Oxford, a structured summary of the critically appraised paper is prepared during the journal club and then added to a computer database in the library. This 
helps the journal club focus on the clinical problem and produce a clinical 'bottom-line'.

Access to high quality sources of evidence is crucial to the feasibility of EBM. As well as improving the sources of evidence themselves (by improving MEDLINE, expanding the number of subjects covered in the Cochrane Library etc.), there is a need to facilitate access to them. One strategy is the production of journals of secondary publication which search large numbers of clinical journals, select the best quality articles using explicit criteria and produce easily assimilable structured abstracts. These enable the clinician to keep up with current advances much more efficiently than attempting to read all the important papers themselves (always a difficult task - there are approximately two million articles published annually in the biomedical literature, in over 20,000 journals, and traditional reviews are often unsystematic and biased, with no description of methods $\left.{ }^{16}\right)$. The first two such journals were the American College of Physicians Journal Club and Evidence-Based Medicine - a CD cumulative compilation of the contents is also produced - Best Evidence. Because neither of these publications is aimed specifically at the needs of the mental health specialist, a new journal, Evidence-Based Mental Health will be produced in late 1997/early 1998. This will include abstracts of papers concerning therapy, diagnosis, prognosis and other issues as well as providing a forum for the development of the application of EBM to the field of mental health.

With the advent of these resources, EBM is now feasible for most clinicians. ${ }^{17}$ It augments traditional clinical skills and provides psychiatrists with a practical and efficient approach to maximising their clinical effectiveness and meeting the increasing demands of their clinical work.

\section{References}

l. Sackett DL, Rosenberg WM, Gray JA, Haynes RB, Richardson WS. Evidencebased medicine: what it is and what it isn't. Evidence-Based medicine 1996; 312 71-2.

2. Milne R, Hicks N. Evidence-based purchasing. Evidence-Based medicine 1996; 1: $101-2$

3. Pocock SJ. Clinical trials: a practical approach. Chichester: John Wiley, 1983. 4. Chalmers I, Haynes B. Reporting, updating, and correcting systematic reviews of the effects of health care. BMJ 1994; 309: 862-5.

5 . Sheldon TA. Research intelligence for policy and practice: the role of the 5. Sheldon TA. Research intelligence for policy and practice: the role of the National Health Service Centre for Reviev

Evidence-Based medicine 1996; $1: 167-8$.
6. Guyatt GH, Sackett DL, Cook DJ. Users' guides to the medical literature. II. How to use an article about therapy or prevention. A. Are the results of the study valid? Evidence-Based Medicine Working Group. JAMA 1993; 270: 2598-601. 7. Guyatt GH, Sackett DL, Cook DJ. Users' guides to the medical literature. II. How to use an article about therapy or prevention. B. What were the results and will they help me in caring for my patients? Evidence-Based Medicine Working Group. JAMA 1994; 271: 59-63.

8. Cook RJ, Sackett DL. The number needed to treat: a clinically useful measure 8. Cook RJ, Sackett DL. The number needed

of treatment effect. BMJ 1995; 310: 452-4. regions. Br J Psychiatry 1992; 160: 621-37.

10 Kendell RE. The College and 'clinical effectiveness'. Psychiatric Bulletin 1997, $21: 385-6$.

11 Geddes JR, Game D, Jenkins NE, Peterson LA, Pottinger GR, Sackett DL What proportion of primary psychiatric interventions are based on randomised evidence? Quality in Health Care 1996; 5: 215-7.

12 Summers A, Kehoe RF. Is psychiatric treatment evidence-based? Lancet 1996; 347: 409-10.

13. Sackett DL, Richardson S, Rosenberg W, Haynes RB. Evidence-based Medicine: How to practise and teach EBM. London: Churchill-Livingstone, 1997.

14. Sackett DL, Haynes RB, Guyatt GH, Tugwell P. Clinical Epidemiology: A 14. Sackett DL, Haynes RB, Guyatt GH, Tugwell P. Clinical Epidemiology: A
basic science for clinical medicine. Boston/Toronto/London: Little, Brown and Company, 1991.

15. Gilbody S. Evidence-based medicine: an improved format for journal clubs. Psychiatric Bulletin 1996; 20: 673-5.

16. Mulrow $C D$. The medical review article: state of the science. Ann Intern Med $1987 ; 106: 485-8$.

17. Geddes JR. Using evidence about clinical effectiveness in everyday clinical practice. Psychiatric Bulletin 1997; 21: 390-3.

\section{Guidelines for Authors}

The fournal's aim is to publish original scientific contributions in psychiatry, psychological medicine (including surgery and obstetrics), and related basic sciences (neurosciences, biological, psychological, and social sciences). Its scope includes any subspecialties of the above, eg. behavioural pharmacology, biological psychiatry, child and adolescent psychiatry, mental handicap. forensic psychiatry, psychotherapies, psychiatry of old age, epidemiology, rehabilitation, psychometrics, substance misuse, sexual studies, linguistics, and the history, philosophy and economics of psychiatry.

The journal will accept original papers, clinical case reports, brief research reports, review articles, perspective articles, historical papers, editorials, practice reviews (medical audits), letters to the editor and book reviews. Review articles are usually invited. Original data papers receive top priority for speedy publication.

Manuscripts should be prepared in accordance with the guidelines of the International Committee of Medical Journal Editors.' The page following the title page should carry an Abstract followed by a list of three to 10 Key Words or short phrases drawn, if possible, from the medical subject headings (MeSH) list of Index Medicus. The Title, Key Words and Abstract should be chosen to help future literature searchers.

The Abstract, up to 150 words for an unstructured or 250 words for the structured abstract, ${ }^{2}$ should state specifically the main purposes, procedures, findings and conclusions of the study, emphasising what is new or important. For original papers, brief research reports, medical audits and review articles, a structured abstract ${ }^{2}$ is required, using the headings Objectives, Methods, Results (Findings for review articles) and Conclusions. Under the Abstract heading of Method, include wherever applicable the study design, setting, patients/participants (selection criteria, description), interventions, observational and analytical methods and main outcome measures. (For review articles specify the methods of literature search and selection). Under the Abstract heading of Results, give the most important specific data together with their statistical significance.

Timely references should highlight the study's relevance to current research or clinical practice. References to journal artides ${ }^{1.3}$ and to books ${ }^{46}$ illustrate the 'Vancouver' style,' with journal titles abbreviated as in Index Medicus.

The Uniform requirements for manuscripts submitted to biomedical journals' has two paragraphs on statistical guidelines. These have been explained and elaborated by Bailar and Mosteller. ${ }^{3}$

Figures and graphs should be clear and of good quality, and should be accompanied by relevant data to facilitate redrawing where necessary. All materials sent for publication should be accompanied by a covering letter signed by all the authors, and such material will become the property of the journal until, and if, publication is refused. Material so referred should not be sent elsewhere for publication. One copy of the manuscript should be retained by the author(s) for reference, and four copies of the manuscript and covering letter, one of these being the original, should be sent to:

\section{The Editor,}

Irish Journal of Psychological Medicine,

99 Upper George's Street.

Dun Laoghaire,

Co Dublin.

All contributions are peer-reviewed by three anonymous assessors and, where relevant, by the deputy statistical editor whom authors may contact for help. Assessments will be sent to the corresponding author usually within six weeks. Where revisions are sought prior to publication, authors are advised to return their revision in quadruplicate, incorporating any suggestions which they agree would improve their paper. The covering letter should respond to each comment, numbered, of each assessor, indicating where the revision deals with it, or why the authors disagree or cannot incorporate it. Each assessor will then receive the authors' revision, covering letter and the previous comments of the other assessors. After the assessors' further comments have been received, the senior editors will make the final decision, including priority and time of publication, and the right to style and if necessary shorten material for publication.

\section{References}

I. International Committee of Medical journal Editors. Uniform requirements for manuscripts submitted to biomedical journals. BMJ 1991; 302: 338-41.

2. Haynes RB, Mulrow CD. Huth El, Altman DG, Gardner MI More information abstracts revisited. Ann intern Med 1990; 11 3: 69-76.

3. Bailar JC. Mosteller F. Guidelines for statistical reporting in articles for medical journals. Ann Intern Med 1988 Feb; 108(2): 266-73.

4. Daly LE, Bourke G]. McGilvray J. Interpretation and uses of medical statistics. 4th ed. Oxford: Blackwell Scientific Publications, 1991: 428-31.

5. Gardner MJ. Altman DG, editors. Statistics with confidence - confidence intervals and statistical guidelines. London: British Medical Journal, 1989: 103-5. [Note: British Medical Journal here is the publisher of a book, not the journal BM].] 6. American Psychiatric Association. Diagnostic and statistical manual of menta disorders. 3rd rev ed. Washington DC: American Psychiatric Association, 1987. 


\section{BECAUSE ALCOHOL \\ DEPENDENTS DESERVE}

THE BEST BREAK THEY CAN GET.. 